\title{
Wicking process in a capillary tube: a new zero-order asymptotic solution
}

\author{
O. Bautista ${ }^{1}$, F. Méndez ${ }^{2}$ \& E. Bautista ${ }^{1}$ \\ ${ }^{1}$ SEPI-ESIME Azcapotzalco. Instituto Politécnico Nacional, Mexico \\ ${ }^{2}$ Facultad de Ingeniería, UNAM, Mexico
}

\begin{abstract}
In this work, we have theoretically re-visited the capillary rise process into a circular tube for very short time scales, retaining in this manner, the physical influence of the inertial effects. We use the boundary-layer technique or matched asymptotic expansion procedure in order to treat this singular problem by identifying two appropriate time scales: one short time scale related with inertial effects, $\sigma$, and the other, $\tau$, the large scale which is basically associated with imbibition effects. Considering that the well-known Washburn's law was derived by neglecting the inertial effects, the corresponding solution has a singular behavior for short times, which is reflected by an infinite mass flow rate. Then, for this purpose we derive a zero-order solution which is enough to avoid the singular behavior of the solution. In this manner, the Washburn's solution represents only the external solution only valid for the large time scale $\tau$. The above analytical result is compared with a numerical solution including the case when the contact angle between the meniscus and the inner surface of the capillary tube becomes a dynamic contact angle. On the other hand, the presence of inertial effects can induce oscillations of the imbibition front which are controlled by the dynamic contact angle. Therefore, in the present work we predict a global asymptotic formula for the temporal evolution of the height of the liquid. In order to show the importance of the inertial terms, we present this evolution for different values of the dimensionless parameters involved in the analysis.
\end{abstract}

Keywords: wicking process, inertial effects, singular perturbation, matched asymptotic expansions. 


\section{Introduction}

In recent years, the phenomenon of capillary wicking has strongly stimulated theoretical studies together with experimental evidences to show some peculiar aspects of these complex processes. In real situations, the wetting of a surface is controlled by rates of spreading of a liquid over the substrate and in general, this effect is devoted for very short times before to reach the well-known equilibrium thermodynamics Young's equation, where the surface tension force is exactly balanced with the gravity force. In addition, the movement of the contact line of the liquid front depends strongly on molecular kinetic of the dynamic contact angle. The existing theories and experimental results about the position and velocity of the contact line are not well understood yet. For instance, relevant studies of the spreading of a drop over horizontal and inclined flat plates have been developed to clarify that, in some cases; the macroscopic contact line can be preceded by a precursor film, where the van der Waals forces are not negligible. This idea was originally proposed by De Gennes [1]. Nowadays, an acceptable point of view to treat the dynamic of the contact angle is to include molecular forces, like van der Walls forces, improving in this manner, the hydrodynamics macroscopic models. In this direction, Treviño et al. [2, 3] carried out a theoretical analysis to predict the influence of the precursor film on the dynamics of an axisymmetric drop spreading over a horizontal surface. The state of the art can be found in the book of Middleman [4], where relevant topics and applications are conducted to illustrate different wicking phenomena.

Since the pioneer work of Washburn [5], several mathematical models have been proposed to analyze those cases where the capillary forces always have a predominant effect. In this direction, the classical works of Joos et al. [6] and Batten [7] show rigorously the main forces that act on a liquid rising up a capillary tube. Hamraoui et al. [8] using high-speed imaging technique and solving a particular Washburn-Rideal-Lucas equation based on a fully developed flow, showed the physical influence of the dynamic contact angle on the wicking process. In this study, the authors postulated a fundamental relationship between the dynamic contact angle and the rate of the liquid rise within the capillary tube and the mathematical solution was validated through the experimental results. A similar study was reported by Hamraoui and Nylander [9] including numerical predictions of oscillations for the imbibition front. In reality, these oscillations were previously reported by Quéré [10] if the liquid viscosity is low enough.

In this paper, following the proposed analytical models by Hamraoui et al. [8] and Duarte et al. [11], we present an asymptotic analysis of zero-order to characterize the initial step of the wicking penetration process into a capillary circular tube, for the case when the inertial terms are important. The fundamental idea is to improve the existing theoretical predictions reported by previous schemes and related with the velocity of the front, using singular perturbation techniques. We anticipate that a singular behavior prevails if, for example, inertial and gravity forces are neglected. In this case, the most simple Washburn's law shows that the penetration velocity is of order of 
$U(=d h / d t) \sim t^{-1 / 2}$ (see Ref. [4]) and for values of $t \rightarrow 0$, the velocity $U$ is undetermined. In order to avoid this singularity, which reflects, in addition, an infinite mass flow rate into the capillary tube, we use two time scales to identify the dominant forces. For each time scale, we derive an appropriate zero-order governing equation and the corresponding solutions are matched according a well pre-established singular perturbation technique. Recognizing the importance of the inertial terms for the initial step of the wicking process, we anticipate that is only sufficient to derive a zero-order global solution, correcting up to this order the singular behavior of the Washburn's law. Therefore, in this first part of the present work the associated corrections with higher-order terms are omitted for simplicity.

\section{Theoretical analysis}

The present model corresponds to eqn. (1a) from Hamraoui et al. [8], and the main forces that act on a liquid rising up a capillary tube are due to surface tension, gravity, viscosity and inertia, respectively:

$$
2 \pi R \gamma\left(1-\frac{\beta}{\gamma} \frac{d h}{d t}\right)=\pi R^{2} \rho g h+8 \pi \mu h \frac{d h}{d t}+\pi R^{2} \rho \frac{d}{d t}\left(h \frac{d h}{d t}\right)
$$

where $R$ is the radius of the capillary, $\beta$ is a constant related with the dynamic contact angle, $h(t)$ is the height of the liquid at time $t . \gamma, \rho, \mu$ are the surface tension, density and dynamic viscosity of the liquid, respectively, and $g$ is the acceleration due to gravity force. In order to derive the above equation were basically neglected the entrance hydrodynamic effects and the liquid was assumed Newtonian, then we can write the average velocity of liquid rising at the capillary from Poiseuille's law as $U=d h / d t=R^{2} \Delta P / 8 \mu h$. In addition, we adopt the relationship between dynamic contact angle, $\theta_{d}$ and the rate $d h / d t$ given by $\cos \theta_{d}(t)=1-(\beta / \gamma)(d h / d t)$. The details of the above considerations can be found elsewhere, [8]. In this form, the present forces included in eqn. (1) are expressed in terms of the unknown height $h(t)$. This scheme has widely been used in lubrication theory to analyze the fluid flow in thin-liquid films (Oron et al. [12]). The above non-linear ordinary differential equation must satisfy two initial conditions. Traditionally, the majority of the published works only include the first of these initial conditions. The reason is based on neglecting the inertia terms. However, for very short times, the inertial terms must be included, even more for those cases of large radius. Therefore, we propose the following initial conditions:

$$
t=0: h=0 \text { and } \frac{d h}{d t}=Q .
$$


In the last initial condition, $Q$ is the unknown volumetric flow rate per unit area at the base of the capillary tube and must be determined as a part of the solution. However, in this manner the system of eqns. (1)-(2) is incomplete because $Q$ is unknown. Therefore, it is necessary to add other restriction. This other condition comes from the equilibrium condition of the imbibition front. The above means that there is an equilibrium time, $t_{e q}$, for which we reach the equilibrium height $h_{e q}$ given by $2 \gamma / \rho g R$. This last relationship is readily derived from the balance between the surface tension and gravity forces. Obviously, the equilibrium time $t_{e q}$ must then be determined as a part of the solution. From this point of view, therefore, the additional condition can be written as

$$
t=t_{e q}: h=h_{e q}=\frac{2 \gamma}{\rho g R}
$$

Now, for solving eqn. (1), we use appropriate dimensionless variables taking into account that, in a first approximation, the characteristic time $t_{c}$ of the wicking penetration process is determined by a balance between the surface tension and viscosity forces. This order relationship can be written as $2 \pi R \gamma \sim 8 \pi \mu h_{e q}^{2} / t_{c}$. Introducing the equilibrium height into the above order relationship, we obtain that $t_{c} \sim 16 \mu \gamma / \rho^{2} g^{2} R^{3}$. We assume $t_{c} \sim t_{\text {eq }}$. Therefore, introducing the following dimensionless variables:

$$
\tau=\frac{t}{t_{c}}, Y=\frac{h}{h_{e}}
$$

the system of Equations (1)-(3) are transformed to:

$$
\left(1-\alpha \frac{d Y}{d \tau}\right)=Y+Y \frac{d Y}{d \tau}+\varepsilon \frac{d}{d \tau}\left(Y \frac{d Y}{d \tau}\right),
$$

with

$$
\tau=0: Y=0, \frac{d Y}{d \tau}=\lambda \quad ; \quad \tau=\tau_{e q}: Y=1
$$

and the parameters $\alpha, \lambda$, and $\varepsilon$ are defined as:

$$
\alpha=\frac{\beta \rho g R^{2}}{8 \mu \gamma}, \lambda=\frac{8 \mu Q}{\rho g R^{2}} \text { and } \varepsilon=\frac{\rho g R^{2}}{128 \gamma} \frac{g R^{3} \rho^{2}}{\mu^{2}}=\frac{B o G a}{128}
$$


where $B o$ and $G a$ represent the Bond and Galileo numbers, respectively. Therefore, the solution of the problem (5)-(6) shall provide $Y=Y\left(\tau, \tau_{e q}, \alpha, \lambda, \varepsilon\right)$. In the above relationship $\tau_{\text {eq }}$ is defined as $\rho^{2} g^{2} R^{3} t_{e q} / 16 \mu \gamma$ with $t_{e q}$ unknown. Therefore, the set of eqns. (5)-(6) represent an eigenvalue problem because the time $t_{e q}$ is unknown. In this manner, the solutions are constructed by taking into account that for arbitrary values of the dimensionless parameters $\alpha, \lambda$ and $\varepsilon$, we must obtain a unique solution of $Y=Y\left(\tau, \tau_{e q}, \alpha, \lambda, \varepsilon\right)$ for each value of the equilibrium time $\tau_{e q}$. In the remainder of this paper we analyze and classify the solutions according to the assumed values of $\alpha$, taking advantage of the fact that in general, $\alpha$ and $\varepsilon$ are very small compared with unity. We anticipate that the values of $\lambda$ are irrelevant for the zero-order solution of $Y$.

\subsection{Asymptotic limit of $\alpha \ll 1$ (pre-wetting surface)}

As we are concerned, a first approximation, to clarify the role of the inertial terms we have omit in the present section, the physical influence of the dynamic contact angle $\theta_{d}$. This particular situation can occur, for instance, when the internal surface in contact with the liquid has been previously wetted. Thus, we have that for $\alpha \ll 1$ the dynamic contact angle, $\theta_{d}$, practically coincides with of the equilibrium or static angle, $\theta_{e q}$. For simplicity, we take $\theta_{e q} \sim 0$, which is a good approximation for wetted surfaces. However, we emphasize that this particular restriction does not significantly affect the general validity of the present analysis. Indeed, for $\theta_{e q} \neq 0$ the first term of the right-hand side of eqn.

(5) can be easily modified by a constant factor given by $\cos \left(\theta_{e}\right)$. Thus, eqn. (5) reduces to:

$$
1=Y+Y \frac{d Y}{d \tau}+\varepsilon \frac{d}{d \tau}\left(Y \frac{d Y}{d \tau}\right) .
$$

The numerical values for $\varepsilon$ are generally small and therefore, the above equation dictates that the inertial terms are only important for a time scale of the order of $\tau \sim \varepsilon$. Otherwise, for $\tau \sim 1$, the inertial term represented by the last term of the right-hand side of the above equation is negligible, in a first approximation. Thus, we can introduce two time scales to study the problem.

\subsubsection{Formulation for large times $(\tau \sim 1)$}

For this relevant limit, we propose the following expansion

$$
Y=Y_{0}+\varepsilon Y_{1}+O\left(\varepsilon^{2}\right)
$$


and substituting into eqn. (5), by retaining only terms of zero order, we obtain that

$$
1=Y_{0}+Y_{0} \frac{d Y_{0}}{d \tau},
$$

and the solution is readily derived and given by (undetermined only by the constant $C_{0}$ ):

$$
1-Y_{0}-\operatorname{Ln}\left(1-Y_{0}\right)=\tau+C_{0} .
$$

In principle, this solution can not satisfy the initial condition $Y(\tau=0)=0$, because the inertial terms were neglected. Therefore, in order to evaluate the constant $C_{0}$, we must retain the inertial terms. We show that is necessary to use a small time scale of order $\tau \sim \varepsilon$ consider the influence of the inertial effects.

\subsubsection{Formulation for short times $(\tau \sim \varepsilon)$}

In this case, we accept that for short times the dominant terms of eqn. (8) are basically controlled by the surface tension and inertial forces. In this manner, the suitable scales to analyze this initial step are $\sigma=\tau / \varepsilon \sim O(1)$ and $y(\sigma)=Y / \sqrt{\varepsilon} \sim O(1)$ and eqn. (8) is transformed to:

$$
\frac{d}{d \sigma}\left(y \frac{d y}{d \sigma}\right)+y \frac{d y}{d \sigma}+\varepsilon^{1 / 2} y=1 .
$$

Now, in order to find the zero-order solution of this equation, we propose the following expansion:

$$
y=y_{0}+O(\varepsilon)
$$

and the leading order equation is governed by a balance between the inertia, viscosity and surface tension forces, given by

$$
\frac{d}{d \sigma}\left(y_{0} \frac{d y_{0}}{d \sigma}\right)+y_{0} \frac{d y_{0}}{d \sigma}=1,
$$

with the initial conditions:

$$
\sigma=0: y_{0}=0 \text { and } \frac{d y_{0}}{d \sigma}=\sqrt{\varepsilon} \lambda \sim O(1) .
$$

The non-linear differential equation (14) of second order can be easily solved because admits a first integral. The resulting first order non-linear equation is solved by using an appropriate integrating factor; for simplicity the details are 
omitted. Following the above procedure and applying the initial conditions (15), we obtain:

$$
y_{0}=\sqrt{2 \sigma+2[\exp (-\sigma)-1]}
$$

It is very important to note that the asymptotic solution for short times $\sigma \rightarrow 0$ is $y_{0} \sim \sigma(1-\sigma / 6)+\ldots$. The same asymptotic formula was previously derived by Quéré [10] using other formulation for modeling the initial steps, where predominate inertial and surface tension effects. Thus, for short times the rising of the capillary wicking has a linear behavior, which avoids that the velocity displays a singular behavior. In addition, the above asymptotic relationship is independent of the dimensionless mass rate $\lambda$.

Retaining then the inertial terms in order to obtain a global solution valid for all time, we can use the well-known asymptotic matching technique, which can be found elsewhere, (Van Dyke, [13]). In this case, we construct a global solution given by,

$$
Y=Y_{0}(\tau)+\sqrt{\varepsilon} y_{0}(\sigma)-Y_{\text {match }},
$$

where $Y_{\text {match }}$ represents the intermediate solution valid in the matching region $\varepsilon<\tau<1$. In this zone, the solutions have a similar behavior and can be calculated by using the following matching asymptotic principle [13],

$$
Y_{\text {match }} \sim \underset{\lim \tau \rightarrow 0}{Y}(\tau) \sim \underset{\lim \sigma \rightarrow \infty}{\sqrt{\varepsilon} y}(\sigma) .
$$

Thus, applying this condition to the solutions (11) and (16) and expanding adequately both solutions, can be easily shown that the constant $C_{0}=1$. In this form, the zero-order global solution is based effectively on an intermediate region $\varepsilon<\tau<1$, where the short and large times solutions are equivalent. This matching solution is given as $Y_{\text {match }}=\sqrt{2 \tau}$ and the global solution with the aid of the above comments is:

$$
Y=\sqrt{2 \tau+2 \varepsilon[\exp (-\tau / \varepsilon)-1]}+Y_{0}-\sqrt{2 \tau},
$$

where $Y_{0}$ is the implicit solution of:

$$
Y_{0}+\operatorname{Ln}\left(1-Y_{0}\right)=-\tau
$$

The above transcendental equation for $Y_{0}(\tau)$ has an approximate analytical solution. We follow the approach presented elsewhere (Fries and Dreyer [14]). The solution for eqn. (20) is given by 


$$
Y_{0}=1+W\left(-e^{-1-\tau}\right)
$$

where $W$ is the Lambert's function. Finally, substituting eqn. (21) into eqn. (19), we obtain that

$$
Y=\sqrt{2 \tau+2 \varepsilon[\exp (-\tau / \varepsilon)-1]}+1+W\left(-e^{-(1+\tau)}\right)-\sqrt{2 \tau},
$$

which is a global analytic solution for $Y(\tau)$. It is really important to note that in order to calculate the numerical value of the Lambert function $W$ that appears on eqns. (21)-(22), we use an approximated function previously developed [14], with a maximum relative error of $0.1 \%$ and given by the relationship

$$
W\left(-e^{-(1+\tau)}\right) \sim-1+\frac{\sqrt{2\left(1-e^{-\tau}\right)}}{1+\frac{4.13501 \sqrt{2\left(1-e^{-\tau}\right)}}{12.7036+\sqrt{2\left(1-e^{-\tau}\right)}}},
$$

valid for $-e^{-1} \leq-e^{-1-\tau} \leq 0$, where $e$ is the well-known Euler's number given by $e=2.718281 \ldots$

\subsection{Numerical scheme}

In this subsection, we present some details related with the numerical procedure to complete the solutions of eqns. (5)-(6) for values of $\alpha \neq 0$. We have included these numerical estimations to compare with the asymptotic solution of zero-order. The class of governing equation given by eqn. (5) can be readily integrated by the classical Runge-Kutta method of fourth-order. In our case, we can define the following variables

$$
Y=\varphi_{1} \quad \text { and } \quad \frac{d Y}{d \tau}=\varphi_{2},
$$

and the second-order nonlinear differential equation (5) with the aid of the above relationships can be transformed to system of two first-order equations given by

$$
\begin{aligned}
& \frac{d \varphi_{1}}{d \tau}=\varphi_{2} \\
& \frac{d \varphi_{2}}{d \tau}=\frac{1-\varphi_{1}-\left(\alpha+\varphi_{1}\right) \varphi_{2}-\varepsilon \varphi_{2}^{2}}{\varepsilon \varphi_{1}} .
\end{aligned}
$$

It is well-known that this method requires initial conditions to begin the first iterations. In our case and from eqn. (6), the part that only corresponds to the initial conditions is provided by $Y(0)=0$ and $d Y(0) / d \tau=\lambda$, which can be 
rewritten as $\varphi_{1}(0)=0$ and $\varphi_{2}(0)=\lambda$. The use of the above initial conditions yields a divergent behavior for both functions $\varphi_{1}$ and $\varphi_{2}$. Therefore, the numerical procedure to integrate eqns. (25) is to replace the above initial conditions by the asymptotic relationships for short times derived in section 2.1.2. In terms of the functions $\varphi_{1}$ and $\varphi_{2}$, the initial conditions are the following

$$
\varphi_{1}(0) \sim \varphi_{1}(\Delta \tau \rightarrow 0)=\frac{\Delta \tau}{\varepsilon^{1 / 2}}\left(1-\frac{\Delta \tau}{\varepsilon}\right)+O\left[\frac{(\Delta \tau)^{3}}{\varepsilon^{5 / 2}}\right]
$$

and

$$
\varphi_{2}(0) \sim \varphi_{2}(\Delta \tau \rightarrow 0)=\frac{1}{\varepsilon^{1 / 2}}\left(1-\frac{\Delta \tau}{3 \varepsilon}\right)+O\left[\frac{(\Delta \tau)^{2}}{\varepsilon^{5 / 2}}\right] .
$$

We can appreciate that for finite and small values of the time increment, $\Delta \tau$, the initial condition (26) remains always different to zero and the other initial condition, given by eqn. (27), depends only the parameter $\varepsilon$, which assumes finite values in our numerical essays. In the present estimations, we use $\Delta \tau=0.00001$ and different values of the parameter $\varepsilon$. In this manner the divergence is eliminated numerically.

\section{Results and conclusions}

In this paper we developed a singular asymptotic analysis to describe the initial steps of the temporal evolution of wicking penetration of a fluid into a capillary tube. The use of two time scales permits us to conduct a boundary layer analysis to clarify the physical role of the inertial terms. In such case, the singularity of the front velocity is eliminated. On the other hand, the present analysis serve to emphasize that the inertia terms are also important not only for a short time scale as we comment from the analytical results, lines below. In this direction, we see that for different substances the inertia effect has a relevant influence even for large times. This occurs as a consequence of the dimensionless parameters $\alpha$ and $\varepsilon$. In Fig. 1, we have plotted the dimensionless height of the wicking front as a function of the dimensionless time for the case of $\alpha=0$ and two different values of the parameter $\varepsilon ; i . e$., $\varepsilon=0$ and $\varepsilon=0.05$, that correspond to the inertial and non-inertial cases, respectively. We see the influence of the inertial terms is to decrease the temporal growth of the liquid's front for small values of the dimensionless parameter $\varepsilon$. Therefore, the asymptotic analysis confirms that the inertial terms always modulate the initial steps of the wicking penetration process. This is an acceptable result always that we do not taken into account time scales smaller than $\varepsilon$, because in this case, capillary penetration can be controlled by hydrodynamic instabilities and more complex phenomena related with the influence of precursor film, chemical 
adsorption activity in the inner surface of capillary tube, intermolecular forces, etc. In addition, the asymptotic solution dictated by eqn. (22) confirms that the usage of the singular perturbation analysis, the zero-order solution is enough to describe the main physical characteristics of the problem.

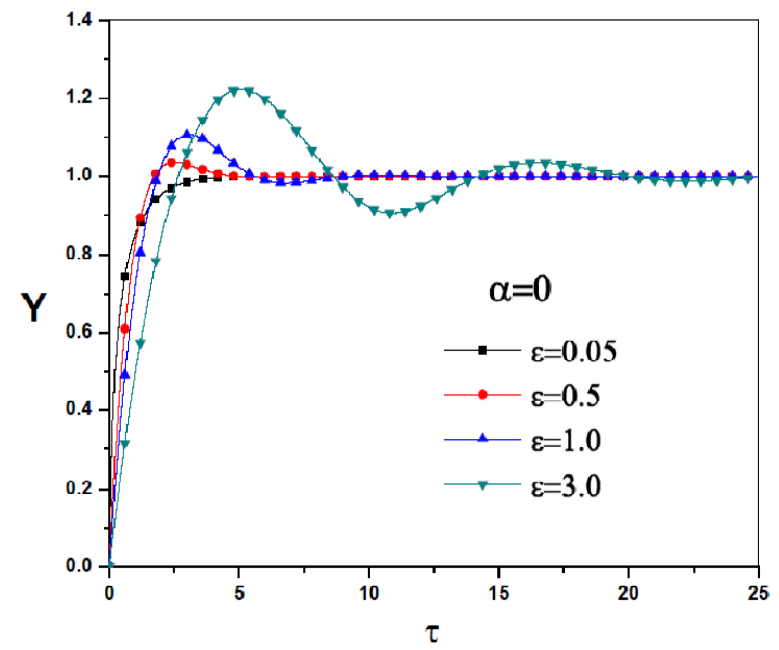

Figure 1: Evolution of capillary rise, for $\alpha=0$.

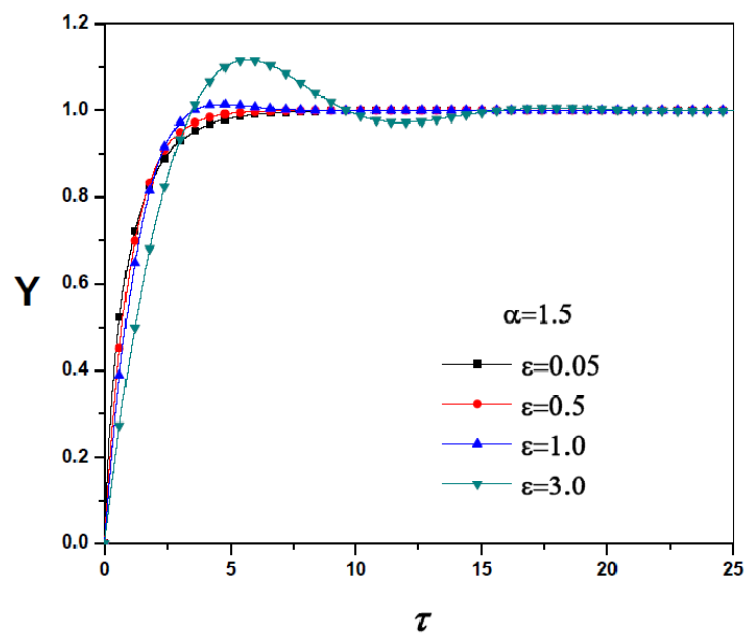

Figure 2: Evolution of capillary rise, for $\alpha=1.5$.

In Fig. 2, we have shown the combined influence of dimensionless parameters $\alpha$ and $\varepsilon$. For instance, in this figure, we use four different values of the parameter $\varepsilon(=0.05,0.5,1.0,3.0)$ and $\alpha=1.5$ to plot the dimensionless 


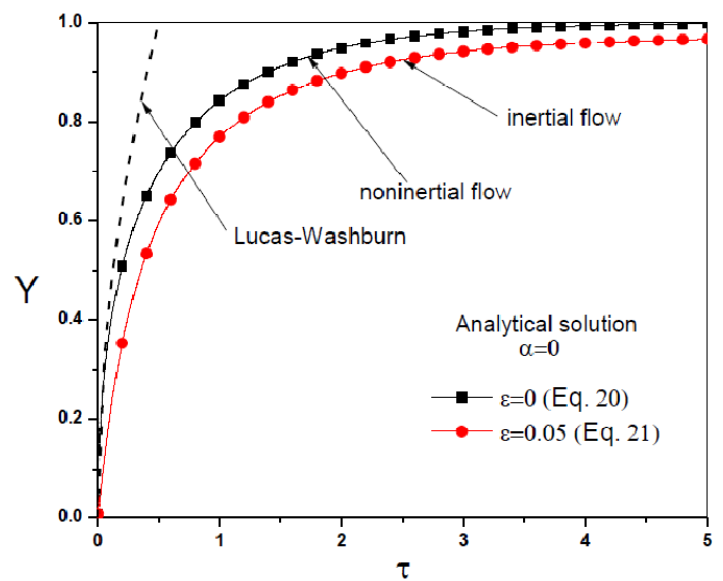

Figure 3: Comparison between the Lucas-Washburn's formula, the present asymptotic zero-order solution, Eq. (22), and the non-inertial solution $(\varepsilon=0)$.

height of the imbibition front as a function of the dimensionless time. Clearly, parameter $\alpha$ works as a dissipative effect because causes a slower wicking process. Therefore, the well-known oscillating pattern previously reported by different authors $[10,11]$ is modulated by the influence of the parameter $\alpha$. Thus, for increasing values of this parameter is easier to reach the equilibrium height of the imbibition. In this sense, the limiting factor above is controlled by the parameter $\varepsilon$. The Fig. 3 shows the difference between the Washburn's law and the obtained in the present work, showing that the evolution of the imbibition front is strongly influenced by considering the inertial effects in the dynamic equation describing the capillary process.

\section{Acknowledgements}

This work has been supported by the research Grant No. 58817 by Consejo Nacional de Ciencia y Tecnología anf 201000375 of Instituto Politécnico Nacional at Mexico.

\section{References}

[1] De Gennes, P. G., Wetting: statics and dynamics. Rev. Mod. Phys. Rev., (57), pp. 827-863, 1985.

[2] Treviño, C., Ferro-Fontán, C., Méndez, F., Asymptotic analysis of axisymmetric drop spreading. Phys. Rev. E., (58), pp. 4478-4484, 1998.

[3] Treviño, C., Méndez, F., Ferro-Fontán, C., Influence of the aspect ratio of a drop in the spreading process over a horizontal surface. Phys Rev. E., (58), pp. $4473-4477,1998$. 
[4] Middleman, S., 1995. Capillary penetration dynamics (Chapter 8). Modeling Axisymmetric Flows, Academic Press, Inc., New York, pp. 211239, 1995.

[5] Washburn, E. W., The dynamics of capillary flow. Phys. Rev., (17), pp. 273-283. 1921.

[6] Joos, P., van Remoortere, P., Bracke, M., The kinetics of wetting in a capillary. J. Coll. Int. Sci., (136), pp. 189-197, 1990.

[7] Batten Jr., G. L., Liquid imbibition in capillaries and packed beds. J. Coll. Int. Sci., (102), pp. 513-518, 1984.

[8] Hamraoui, A., Thuresson, K., Nylander T., Yaminsky, V., Can a dynamic contact angle be understood in terms of a friction coefficient? J. Coll. Int. Sci., (226), pp. 199-204, 2000.

[9] Hamraoui, A., Nylander, T., Analytical approach for the Lucas-Washburn equation. J. Coll. Int. Sci., (250), pp. 415-421, 2002.

[10] Quéré, D., Inertial capillarity. Europhys. Lett., (39), pp. 533-538, 1997.

[11] Duarte, A. A., Strier, D. E., Zanette, D. H., The rise of a liquid in a capillary tube revisited: A hydrodynamical approach. Am. J. Phys., (64), pp. 413-418, 1996.

[12] Oron, A., Davis S. H., Bankoff, S. G., Long-scale evolution of thin liquid films. Rev. Mod. Phys., (69), pp. 931-980, 1997.

[13] Van Dyke, M., Perturbation Methods in Fluid Mechanics, second ed. Academic Press, Inc., New York, 1964.

[14] Fries, N., Dreyer, M., An analytic solution of capillary rise restrained by gravity. J. Coll. Int. Sci., (320), pp. 259-263, 2008.

[15] Corless, R. M., Gonnet, G. H., Hare, D. E. G., Jeffrey D. J., Knuth, D. E., On the Lambert $W$ function. Adv. Comp. Math. (5), pp. 329-359, 1996. 